\title{
Simple Models of Magnetism
}

\author{
Ralph Skomski \\ Department of Physics and Astronomy \\ and \\ Nebraska Center for Materials and Nanoscience \\ University of Nebraska
}




\section{Contents}

List of abbreviations xii

List of panels and tables xiv

Preface xv

1 Introduction: The simplest models of magnetism 1

1.1 Field and magnetization 2

1.2 The circular-current model $\quad 4$

$\begin{array}{ll}1.3 & \text { Paramagnetic spins }\end{array}$

1.4 Ising model and exchange 8

1.5 The viscoelastic model of magnetization dynamics 10

Exercises 13

2 Models of exchange $\quad 15$

$\begin{array}{lll}2.1 & \text { Atomic origin of exchange } & 17\end{array}$

$\begin{array}{lll}\text { 2.1.1 One-electron wave functions } & 18\end{array}$

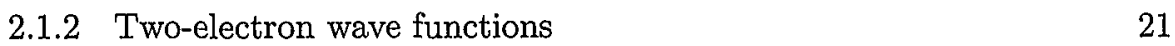

2.1.3 Hamiltonian and spin structure $\quad 22$

$\begin{array}{ll}2.1 .4 & \text { Heisenberg model } \\ 2.1 .5 & 25\end{array}$

2.1.5 Independent-electron approximation 27

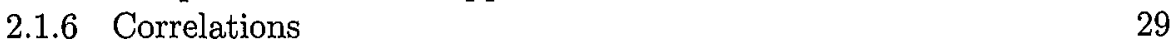

2.1.7 *Hubbard model $\quad 32$

2.1.8 *Kondo model 34

2.2 Magnetic ions 36

2.2.1 Atomic orbitals 36

$\begin{array}{ll}2.2 .2 & \text { Angular-momentum algebra }\end{array}$

2.2.3 Vector model and Hund's rules 41

2.2.4 Spin and orbital moment 41

2.3 Exchange between local moments 44

2.3.1 Exchange in oxides 44

2.3.2 Ruderman-Kittel exchange 46

2.3.3 Zero-temperature spin structure $\quad 48$

2.4 Itinerant magnetism 51

2.4.1 Free electrons, Pauli susceptibility, and the

$\begin{array}{lll}2.4 .2 & \text { Band structure } & 58\end{array}$ 
2.4.3 Stoner model and beyond

2.4.4 *Itinerant antiferromagnets 66

Exercises

3 Models of magnetic anisotropy $\quad 73$

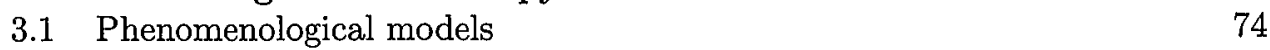

3.1.1 Uniaxial anisotropy 75

3.1.2 Second-order anisotropy of general symmetry 76

3.1.3 Higher-order anisotropies of nonuniaxial symmetry 78

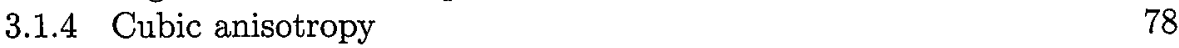

$\begin{array}{ll}3.1 .5 & \text { Anisotropy coefficients }\end{array}$

3.1.6 Anisotropy fields 80

3.2 Models of pair anisotropy $\quad 80$

3.2.1 Dipolar interactions and shape anisotropy 81

3.2.2 Demagnetizing factors $\quad 82$

3.2.3 Applicability of the shape-anisotropy model 83

3.2.4 The Néel model 83

3.3 Spin-orbit coupling and crystal-field interaction 84

3.3.1 Relativistic origin of magnetism 85

3.3.2 Hydrogen-like atomic wave functions $\quad 87$

$\begin{array}{ll}\text { 3.3.3 Crystal-field interaction } & 87\end{array}$

3.3.4 Quenching 89

3.3.5 Spin-orbit coupling 90

3.4 The single-ion model of magnetic anisotropy 91

3.4.1 Rare-earth anisotropy 91

3.4.2 Point-charge model 95

3.4.3 The superposition model 97

3.4.4 Transition-metal anisotropy 98

$\begin{array}{lll}3.5 & \text { Other anisotropies } & 100\end{array}$

3.5.1 Magnetoelasticity 100

3.5.2 Anisotropic exchange 101

3.5.3 Models of surface anisotropy 102

$\begin{array}{ll}\text { Exercises } & 104\end{array}$

4 Micromagnetic models 107

$\begin{array}{lll}4.1 & \text { Stoner-Wohlfarth model } & 110\end{array}$

4.1.1 Aligned Stoner-Wohlfarth particles 111

4.1.2 Angular dependence 112

4.1.3 Spin reorientations and other first-order transitions 113

4.1.4 Limitations of the Stoner-Wohlfarth model 115

$\begin{array}{lll}4.2 \text { Hysteresis } & 116\end{array}$

4.2.1 Micromagnetic free energy 117

4.2.2 *Magnetostatic self-interaction 118

4.2.3 *Exchange stiffness 119

4.2.4 Linearized micromagnetic equations 120

4.2.5 Micromagnetic scaling 122

4.2.6 Domains and domain walls 123 
$\begin{array}{llr}4.3 & \text { Coercivity } & 128\end{array}$

$\begin{array}{lll}4.3 .1 & \text { Nucleation } & 130\end{array}$

$\begin{array}{lll}\text { 4.3.2 Pinning } & 135\end{array}$

4.3.3 Phenomenological coercivity modeling 139

4.4 Grain-boundary models $\quad 141$

$\begin{array}{lll}\text { 4.4.1 Boundary conditions } & 141\end{array}$

$\begin{array}{ll}\text { 4.4.2 Spin structure at grain boundaries } & 143\end{array}$

$\begin{array}{ll}\text { 4.4.3 Models with atomic resolution } & 144\end{array}$

$\begin{array}{ll}\text { 4.4.4 Nanojunctions } & 145\end{array}$

$\begin{array}{ll}\text { Exercises } & 146\end{array}$

$5 \quad$ Finite-temperature magnetism $\quad 149$

$\begin{array}{lll}5.1 \text { Basic statistical mechanics } & 150\end{array}$

$\begin{array}{lll}\text { 5.1.1 Probability and partition function } & 152\end{array}$

$\begin{array}{ll}\text { 5.1.2 * Fluctuations and response } & 153\end{array}$

$\begin{array}{lll}5.1 .3 & \text { Phase transitions } & 155\end{array}$

$\begin{array}{ll}\text { 5.1.4 Landau theory } & 156\end{array}$

5.2 Spin-Space modeling $\quad 159$

$\begin{array}{ll}\text { 5.2.1 Heisenberg models } & 160\end{array}$

5.2.2 Ising, XY, and other $n$-vector models 161

5.2.3* Other discrete and continuum spin models 162

$\begin{array}{lll}5.2 .4 & \text { Ionic excitations } & 163\end{array}$

5.2.5 Spin fluctuations in itinerant magnets 164

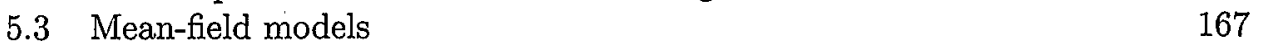

5.3.1 Mean-field Hamiltonians 168

$\begin{array}{ll}\text { 5.3.2 Basic mean-field predictions } & 169\end{array}$

$\begin{array}{ll}\text { 5.3.3* Ornstein-Zernike correlations } & 171\end{array}$

5.3.4 Magnetization and Curie temperature 172

5.3.5 *Mean-field Curie temperature of $n$-vector models 173

$\begin{array}{ll}\text { 5.3.6 Two-sublattice magnetism } & 174\end{array}$

$\begin{array}{ll}\text { 5.3.7 Merits and limitations of mean-field models } & 178\end{array}$

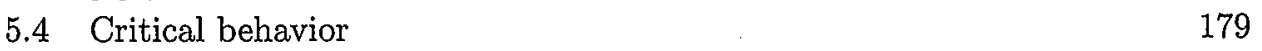

5.4.1 One-dimensional models 180

$\begin{array}{ll}\text { 5.4.2 Superparamagnetic clusters } & 181\end{array}$

$\begin{array}{ll}5.4 .3 * \text { *inzburg criterion } & 183\end{array}$

$\begin{array}{ll}\text { 5.4.4 Fluctuations and criticality } & 184\end{array}$

$\begin{array}{ll}\text { 5.4.5 Renormalization group } & 187\end{array}$

5.5 Temperature dependence of anisotropy 190

$\begin{array}{ll}\text { 5.5.1 Callen and Callen model } & 191\end{array}$

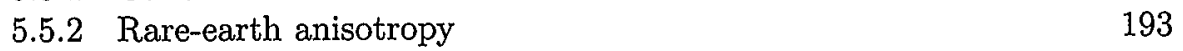

$\begin{array}{ll}\text { 5.5.3 Sublattice modeling } & 195\end{array}$

$\begin{array}{ll}\text { Exercises } & 196\end{array}$

6 Magnetization dynamics 199

6.1 Quantum dynamics and resonance 199

$\begin{array}{ll}\text { 6.1.1 Spin precession } & 201\end{array}$

$\begin{array}{ll}\text { 6.1.2 Uniform magnetic resonance } & 202\end{array}$ 
$\begin{array}{ll}\text { 6.1.3 Spin waves } & 203\end{array}$

6.1.4 Spin dynamics in inhomogeneous magnets* 206

6.2 Relaxation 208

$\begin{array}{ll}\text { 6.2.1 Damped precession } & 209\end{array}$

$\begin{array}{ll}6.2 .2 * \text { Physical origin of relaxation } & 210\end{array}$

$\begin{array}{ll}6.2 .3 * \text { A mechanical model } & 211\end{array}$

6.3 Coarse-grained models 213

$\begin{array}{lll}\text { 6.3.1 Master equation } & 214\end{array}$

$\begin{array}{lll}\text { 6.3.2 Fokker-Planck equations } & 216\end{array}$

$\begin{array}{ll}\text { 6.3.3 Langevin models } & 218\end{array}$

6.4 Slow magnetization dynamics 220

6.4.1 Magnetic viscosity and sweep-rate dependence 223

6.4.2 Superposition model of magnetic viscosity 223

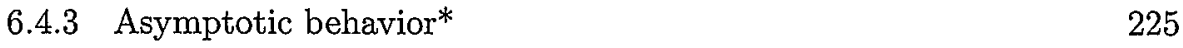

6.4.4 Energy-barrier models 226

$\begin{array}{ll}\text { 6.4.5 *Linear and other laws } & 227\end{array}$

$\begin{array}{lll}6.4 .6 & \text { Superparamagnetism } & 228\end{array}$

$\begin{array}{ll}6.4 .7 * \text { Fluctuations } & 229\end{array}$

Exercises 233

7 Special topics and interdisciplinary models 237

$\begin{array}{lll}7.1 & \text { Disordered magnets and spin glasses } & 237\end{array}$

7.1.1 Atomic disorder and electronic structure 238

$\begin{array}{ll}7.1 .2 * \text { * Green Functions } & 239\end{array}$

7.1.3 Ferromagnetic order in inhomogeneous magnets 242

$\begin{array}{ll}\text { 7.1.4 Spin glasses } & 244\end{array}$

$\begin{array}{lll}7.2 & \text { Soft matter, transport, and magnetism } & 247\end{array}$

7.2.1 Random walks, polymers, and diffusion 248

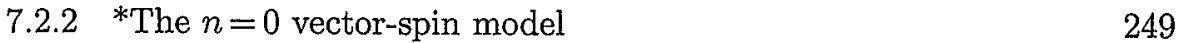

$\begin{array}{lll}7.2 .3 & \text { Polymers and critical dimensionality } & 250\end{array}$

$\begin{array}{lll}7.2 .4 & \text { Percolation } & 252\end{array}$

$\begin{array}{ll}7.2 .5 & \text { Diffusive transport }\end{array} 255$

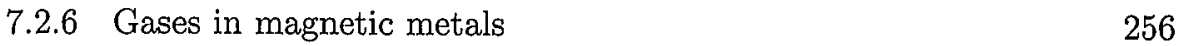

$\begin{array}{ll}\text { 7.2.7 Magnetoresistance } & 258\end{array}$

7.2.8 Other transport phenomena involving magnetism 261

$\begin{array}{lll}7.3 & \text { Bruggeman model } & 263\end{array}$

7.3.1 Static and dynamic properties 263

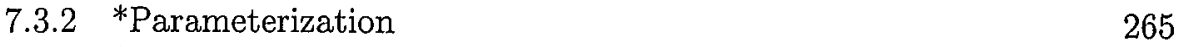

7.3.3 *Self-consistent materials equations 266

$\begin{array}{ll}7.3 .4 * \text { The response parameter } g & 267\end{array}$

7.3.5 *Percolation in the Bruggeman model 267

7.4 Nanostructures, thin films, and surfaces 268

$\begin{array}{lll}\text { 7.4.1 Length scales in nanomagnetism } & 270\end{array}$

$\begin{array}{ll}\text { 7.4.2 Nanomagnetic effects of atomic origin } & 271\end{array}$

$\begin{array}{lll}\text { 7.4.3 Random anisotropy } & 274\end{array}$ 
$\begin{array}{ll}\text { 7.4.4 } * \text { Cooperative magnetization processes } & 277\end{array}$

$\begin{array}{lll}7.4 .5 & \text { Two-phase nanostructures } & 279\end{array}$

$\begin{array}{ll}7.5 & \text { Beyond magnetism }\end{array} 282$

$\begin{array}{lll}\text { 7.5.1 Metallurgy } & 283\end{array}$

$\begin{array}{ll}7.5 .2 & \text { Biology and medicine }\end{array} 285$

$\begin{array}{lll}\text { 7.5.3 Social sciences } & 286\end{array}$

$\begin{array}{ll}\text { Exercises } & 286\end{array}$

$\begin{array}{ll}\text { Appendix } & 289\end{array}$

$\begin{array}{lll}\text { A.1 Units and constants } & 289\end{array}$

A.1.1 Units systems and notation 289

$\begin{array}{ll}\text { A.1.2 Unit conversions } & 290\end{array}$

A.1.3 Physical constants 290

$\begin{array}{lll}\text { A.2 Mathematics } & 290\end{array}$

$\begin{array}{ll}\text { A.2.1 Linear equations } & 290\end{array}$

$\begin{array}{ll}\text { A.2.2 Eigenmode analysis } & 292\end{array}$

A.2.3 Real $2 \times 2$ matrices $\quad 293$

A.2.4 Vector and functional calculus $\quad 295$

$\begin{array}{ll}\text { A.2.5 Useful formulae } & 297\end{array}$

A.3 Basic quantum mechanics $\quad 297$

$\begin{array}{ll}\text { A.3.1 Time dependence } & 298\end{array}$

A.3.2 Eigenvalues and eigenfunctions 298

$\begin{array}{ll}\text { A.3.3 Perturbation theory } & 299\end{array}$

$\begin{array}{ll}\text { A.3.4 Quantum statistics } & 300\end{array}$

$\begin{array}{ll}\text { A.3.5 Relativistic quantum mechanics } & 302\end{array}$

$\begin{array}{lll}\text { A.4 Electromagnetism } & 304\end{array}$

$\begin{array}{lll}\text { A.4.1 Maxwells equations } & 304\end{array}$

$\begin{array}{ll}\text { A.4.2 Simple magnetostatic solutions } & 306\end{array}$

A.4.3 Simple dynamic solutions 308

A.5 Magnetic materials $\quad 309$

$\begin{array}{ll}\text { A.5.1 Transition-metal elements and alloys } & 310\end{array}$

$\begin{array}{ll}\text { A.5.2 Magnetic oxides } & 314\end{array}$

$\begin{array}{ll}\text { A.5.3 Rare-earth magnets } & 314\end{array}$

A.6 Forgotten and reinvented $\quad 315$

$\begin{array}{ll}\text { References } & 319\end{array}$

$\begin{array}{ll}\text { Index } & 335\end{array}$ 